hep-th/0509046

\title{
Duality symmetry and the form fields of M-theory
}

\author{
Hisham Sati * \\ Department of Physics \\ and \\ Department of Pure Mathematics \\ University of Adelaide \\ Adelaide, SA 5005, \\ Australia \\ Department of Theoretical Physics \\ Research School of Physical Sciences and Engineering \\ The Australian National University \\ Canberra, ACT 0200 \\ Australia
}

\begin{abstract}
In previous work we derived the topological terms in the M-theory action in terms of certain characters that we defined. In this paper, we propose the extention of these characters to include the dual fields. The unified treatment of the M-theory four-form field strength and its dual leads to several observations. In particular we elaborate on the possibility of a twisted cohomology theory with a twist given by degrees higher than three.
\end{abstract}

${ }^{*}$ E-mail: hisham.sati@adelaide.edu.au

Research supported by the Australian Research Council. 


\section{Introduction}

Interesting global information is encoded in the Maxwell-like rank four field $G_{4}$ of M-theory, which is written locally as $G_{4}=d C_{3}$ where $C_{3}$ is the so-called C-field. So one concrete aim in this direction is to understand the nature of this C-field. Another is to understand Hodge duality that relates $G_{4}$ to its dual $* G_{4}$ in eleven dimensions. There is an analogous question in type II string theory where the fields are grouped into a total field strength containing the fields descending from $G_{4}$, by dimensional reduction, as well as their (ten-dimensional) Hodge duals. This package leads to the description in terms of twisted K-theory [1] [2] 3].

We would like then to ask whether, in analogy to the type II case, we can unify both field strengths in eleven dimensions, namely the fields $G_{4}$ and $* G_{4}$. So we seek a generalized cohomology theory in which the eleven-dimensional fields are unified in the same way that the Ramond-Ramond fields (in the presence of Neveu-Schwarz fields) are unified into (twisted) K-theory. Earlier work [4] [5] 6] with I. Kriz viewed elliptic cohomology as the right setting for type II string theory. The corresponding picture in M-theory leads to the question of whether the theory $\mathcal{M}$ proposed in [7] is new or whether it happens to be one of the known generalized cohomology theories. In [8] we proposed a unified quantization condition on $G_{4}$ and its dual by viewing the pair as components of the same total field strength. So the point we look at in the present paper is the possibility that this total field strength 'lives' in some generalized cohomology theory.

One might argue that the problem can be looked at from the complementary picture of branes. In the same way that one has to talk about branes up to creation of other branes in type II string theory [9], here we ask whether one can talk about M-branes up to creation of other M-branes. While the picture is not precisely analogous, one can say that the existence of the $M 5$-brane automatically requires the existence of the $M 2$-brane, via the Hanany-Witten mechanism or via the dielectric effect. ${ }^{1}$

The supermultiplet $\left(g_{\mu \nu}, \psi_{\mu}, C_{3}\right)$ of eleven-dimensional supergravity [1] is composed of the metric, the gravitino and the C-field. Thus, in its standard formulation, the theory is manifestly duality-nonsymmetric. One can then ask about the role of the dual fields in the theory. One can get a free supersymmetric theory based on the dual 6 -index field $C_{6}$, but the corresponding interacting theory is not consistent [12. There is also a duality-symmetric formulation of eleven-dimensional supergravity [13. ${ }^{2}$ However, such a formulation does not seem to accommodate nontrivial topology or fields that are nontrivial in cohomology. There is also the duality-symmetric formulation of the nongravitational fields in [17], again

\footnotetext{
${ }^{1}$ This was discussed briefly in [10].

${ }^{2}$ For ten-dimensional supergravity theories, this was dicussed in [14], 15] and [16].
} 
assuming $G_{4}=d C_{3}$, i.e. the field $G_{4}$ is trivial in cohomology, $\left[G_{4}\right]=0$.

We need a degree four 'Bott generator' and either a degree seven or a degree eight gnerator for the dual. Using the rank seven field $* G_{4}$ as the dual field, we find the equations of motion (henceforth EOM) and the Bianchi identity as components of a unified expression of the total field strength, using a twisted differential, with the twist now given by the degree four field $G_{4}$ instead of $\mathrm{H}_{3}$, in the usual case of type II string theory. Adding the one-loop term $I_{8}$ to the EOM serves a priori as an obstruction to having such a twisted cohomology. However, by absorbing $I_{8}$ in the definition of the dual field strength one still gets a twisting.

One can ask about the relevance of the $E_{8}$ gauge theory. We know that the degree four field $G_{4}$ is intimately related to $E_{8}$, at least topologically [18. What we are advocating is that there two ways of looking at the problem, one via $E_{8}$, and another via some generalized cohomology theory. But then adding the dual fields, one seems to break that connection, and in this case it seems possible to only look for a generalized cohomology interpretation, as the homotopy type of $E_{8}$ does not allow for a direct interpretation of the dual field(s).

So we argue for two points of view regarding the fields. The first is the bundle picture in which only the lower-rank fields 'electric' fields are described, e.g. $G_{4}$ in M-theory via $E_{8}, F_{2}$ in type IIA via the M-theory $S^{1}$-bundle. The second is the generalized cohomology picture where the field strengths and their duals are grouped into one total field strength that lives in the corresponding generalized cohomology theory, e.g. twisted K-theory for type II. Thus taking the second point of view, the aim of this paper is to argue for a generalized cohomology theory for the case of the M-theory field strength $G_{4}$ and its dual. Such a unification was already started in [8] where the class of $G_{4}$ and the dual class $\Theta$ (realizing the RHS of the EOM) were given a unified expression that reflected their quantization laws.

The existence of the corresponding generalized cohomology theory was proposed in [7] and further properties were given in [8].

\section{The total field strength}

First note that, unlike the RR fields which have mod 2 periodicity, the fields of M-theory do not enjoy such a periodicity. This is obvious because one of the fields has even rank and the other has odd rank. Besides there are only two of them. One can ask first whether there is a Bott element of dimension three (= the difference of the two ranks) that can take the role which the usual Bott element played in type II. The answer is negative and there is no such element in the class of theories descending directly from $M U$. So one can then ask whether there is another way to form a total M-theory field strength with a uniform degree. One is then forced to use more than one element to do the job. Again there is no element of odd 
degree, so in order to be able to say something useful, one seeks a modification of the point of view in which even degree fields are included. But what exactly should we do? Two things come to mind. First we can try to lift to the bounding twelve dimensional theory defined on $Z^{12}$ with $\partial Z^{12}=Y^{11}$. Here, one possibility is then to look at the four/eight combination $G^{(12)}=G_{4}+*_{12} G_{4}$ in twelve dimensions. Then the arguments that hold for $G_{4}+d *_{11} G_{4}$ in eleven dimensions hold for $G^{(12)}$ as well. ${ }^{3}$ Second, we can work with an eight-form in eleven dimensions, that we view as the dual field instead of the seven form. On the other hand, if we insist on working with odd forms, then this seems to suggest some deformation of cohomology rings which involves odd generators.

We are looking for a generator of degree four that makes a degree zero form when multiplied with $G_{4}$. Since $\operatorname{dim} v_{n}=2 p^{n}-2$, there is only one generator of degree four, which the first generator at $p=3$. What theory is a good candidate theory to include this generator? It is possible that this is either of the first Morava K-theories at $p=3$, i.e. either $\widetilde{K}(1)$ or $K(1)$ with coefficient rings $\widetilde{K}(1)_{*}=\mathbb{Z}\left[v_{1}, v_{1}^{-1}\right]$, and $K(1)_{*}=\mathbb{Z} / 3\left[v_{1}, v_{1}^{-1}\right]$, respectively. We can then form the desired class 4

$$
\left(v_{1, p=3}\right)^{-1} G_{4} .
$$

As in the case for $G_{4}$ we are looking for a generator whose degree is the same as the degree of the field, and which is inverted so that its inverse can be used to write down a uniform degree zero field. So here we need a degree eight generator. Now we would like to find an expression of total degree zero for the total M-theory field strength. The desired generator is the square of $v_{1, p=3}$, which has total dimension $4+4=8$. So with this possibility, we can write the following expression for the uniform total field strength ${ }^{5}$

$$
G=\left(v_{1, p=3}\right)^{-1} G_{4}+\left(v_{1, p=3}\right)^{-2} G_{8} .
$$

With this, we are using the same generator for the whole expression, which is the case analogous to the type II situation, One possibility that that we are then dealing with the $p=3$ first (integral) Morava K-theory. One can ask whether the problem can be looked at without specializing to a particular prime. The theory of Topological Modular Forms, $\operatorname{tm} f$, has an interesting feature that it is not localized at a given prime, i.e. is not local and unifies all primes - see $[6$ for a discussion on the relevance of TMF from a different but related point of view. This is attractive, and seems to be what a theory like M-theory should be doing. Besides, this might make sense since the vector bundles (or their 'higher-degree'

\footnotetext{
${ }^{3}$ Throughout the paper, if the Hodge star operator has no explicit dimension label then it refers to the eleven-dimensional one.

${ }^{4}$ In writing this expression and all the analogous ones, we are implicitly tensoring with $\mathbb{R}$ (or $\mathbb{Q}$ ).

${ }^{5}$ This is meant to be analogous to the uniform degree zero expressions of the RR field strengths in [20].
} 
analogs) are real, and TMF is a real theory - it is to elliptic cohomology $E$ as $K O$-theory is to $K$-theory. One can ask whether there are degree four and degree eight generators in tmf, which can be used for the total field strength. Indeed there are such generators, which were used in [6].

As far as dynamics goes, it does not make much sense to talk about $* G_{4}$ or $d * G_{4}$ alone, because their dynamics involve $G_{4}$ (cf. the EOM of $G_{4}$ ). So in order to include the dual picture, one can at best look for a duality-symmetric formulation of the character, i.e. as opposed to a dual description. If we use the eight-form $d * G_{4}$ as the 'dual' form, then the corresponding exponential is

$$
e^{G_{4}+d * G_{4}} .
$$

We ask the question whether from this we can get the EOM and the Bianchi identity. By looking at the degrees of the forms, we see that while we can get the Bianchi identity by looking at the degree five component, i.e.

$$
\left[d\left(e^{G_{4}+d * G_{4}}\right)\right]_{(5)},
$$

we cannot get the EOM, simply because the degrees of forms would not match. ${ }^{6}$

One can then ask whether the exponential (2.3) can be looked at in some other way that would give the EOM and Bianchi. While the EOM can be obtained by some 'flatness condition' on the character, i.e.

$$
\left[e^{G_{4}+d * G_{4}}\right]_{(8)}=0,
$$

the Bianchi identity does not follow. One instead gets a flatness condition on $G_{4}$ as well if one were to look at the degree four component of the expression (2.5). Even though one can say we got both the EOM and the Bianchi identity, we actually did not do that by using the same expression, and this is obviously not satisfactory. This seems to indicate that while the quantization conditions on the forms 8 favors the four/eight combination, the dynamics favors instead the four/seven combinations of field strengths.

Let us now look at the effect of including the generators- let us call them $v$ and $\widetilde{v}$-in (2.3). Doing so results in the expression

$$
\left[\left(e^{v^{-1} G_{4}+\widetilde{v}^{-1} d * G_{4}}\right)\right]_{(8)}=\frac{1}{2} v^{-2} G_{4} \wedge G_{4}+\widetilde{v}^{-1} d * G_{4} .
$$

So requiring that we get the EOM via factoring out the generators leads to the obvious

\footnotetext{
${ }^{6}$ unless the differential does not act on the exponential, which is not what is meant to happen.
} 
condition that ${ }^{7}$

$$
v^{2}=\widetilde{v}
$$

Naturally, we would like to see whether such a condition can occur in the generalized cohomology theories that we consider in this paper. We check the dimensions of the generators. Since in general that dimension at 'level' $n$ and prime $p$ is $\operatorname{dim} v_{n}=2\left(p^{n}-1\right)$, we then need to satisfy the equality

$$
\left[2\left(p^{n}-1\right)\right]^{2}=2\left(p^{m}-1\right),
$$

where $m>n$. Even though such an expression is not expected to have many solutions in general, it is still more general than we want.

It might be desirable to require that the total expression on the RHS of (2.6) have degree zero. It turns out that this is not possible within the current context, and the next best thing is to require the first generator $v$ to have degree four. ${ }^{8}$ This then implies, via $2\left(p^{n}-1\right)=4$, that $p=3$ and $n=1$. Of course the equality is then satisfied and the dimension of $\widetilde{v}$ is 16 with $m=2$ and the same prime $p=3$.

Let us go back and look at what the above implies for the relationship between the dimensions of the generators and the dimensions of the field strengths. In the above we asked whether the expanded exponential expression has total degree zero. But then going back to the exponent, we see that it does not have total degree zero, because we have the generator $\widetilde{v}$, which we found to have dimension sixteen, multiplying $d * G_{4}$ which has rank eight as a form or a class. However, it is still true that the $G_{4}$ part has degree zero. What we learn from this is that what matters is for the degrees of the factors to match after expanding the exponential and not as they stand in the exponent. As mentioned earlier, generators of degree four and eight can be obtained from tmf (cf. [6] ).

\section{$3 \quad$ A twisted (generalized) cohomology?}

In this section, we would like to use the degree seven field as the dual field to $G_{4}$ and thus take the total field strength to be $G=G_{4}+*_{11} G_{4}$. We would like to use such an expression (and slight variations on it -see below) as it is duality-symmetric ${ }^{9}$ in the electric-magnetic

\footnotetext{
${ }^{7}$ It is interesting that if we interpret $v$ and $\widetilde{v}$ as the generators introduced in [17 and used in the next section, then the corresponding statement would be $\{v, v\}=-\widetilde{v}$, i.e. one of the relations of the gauge algebra for $G_{4}$ and $* G_{4}$. The minus sign would then make (2.6) equal to $(d+\mathcal{G})^{2}$, the obstruction to nilpotency.

${ }^{8}$ In any case, even without requiring the $G_{4}$ term to have degree zero, one sees upon inspecting (2.8), at least for relatively low $n, m$ and $p$ (which are the only relevant), that the result of the discussion does not change.

${ }^{9}$ This is meant to be in the sense that the expression contains both $G_{4}$ and its dual $* G_{4}$, and that it is invariant under the exchange $G_{4} \leftrightarrow * G_{4}$. It is not meant to be in the sense of exchanging $G$ and $* G$ as we
} 
or membrane-fivebrane sense. Then it is interesting that one can write the Bianchi identity and the EOM of $G_{4}$, respectively, as the degree five and the degree eight component of the expression

$$
\left(d+\frac{1}{2} G_{4} \wedge+\frac{1}{2} * G_{4} \wedge\right) G=0 .
$$

The degree eleven component, i.e. the cross-terms between $G_{4}$ and $* G_{4}$, vanish because of the relative minus sign, ${ }^{10}$ and the $* G_{4} \wedge * G_{4}$ term vanishes because it involves the same form of odd degree.

There are several interesting aspects to equation (3.1). First, one can ask whether this has the form of some twisted structure in analogy to that associated with the RR fields in type II string theory, where one has for the total field strength $F$,

$$
d F=H_{3} \wedge F
$$

Written as

$$
d_{H_{3}} F=\left(d-H_{3} \wedge\right) F=0,
$$

this leads to interpreting $d-H_{3}$ as the differential in twisted (de Rham) cohomology $H^{*}(X, H ; \mathbb{R})$, even for type IIA and odd for type IIB [3]. One can easily check that $\left(d_{H_{3}}\right)^{2}$ is indeed zero [3], which follows from the fact that the twisting field $H_{3}$ is closed and that the wedge product of two twisting fields $H_{3} \wedge H_{3}$ vanishes just because it is the wedge product of the same differential form of odd degree.

Going back to (3.1), we ask whether an analogous structure appears. Of course we have obvious differences from the type II case: what is to be interpreted as a 'twisting field', $\frac{1}{2} G$, is now part of the total field that is being twisted, namely $G$. ${ }^{11}$ The other difference is that the twist now involves an even rank field, which while it is closed in analogy to $H_{3}$, the wedge of two copies of which does not vanish since it is even-dimensional. If we interpret the combination $d+\frac{1}{2} G_{4}+\frac{1}{2} * G_{4}$ as a new differential $d_{G}$ and hope that it forms a cohomology, then the nilpotency does not seem to be immediately obvious. However, it turns out that the situation is in fact encouraging. To see this, let us simply calculate the action of its square on the total field strength,

$$
d_{G}^{2} G=\left(d+\frac{1}{2} G_{4} \wedge+\frac{1}{2} * G_{4} \wedge\right)^{2} G
$$

will see explicitly later when the generators of the gauge algebra are included.

${ }^{10}$ since $\alpha_{k} \wedge \beta_{l}=(-)^{k l} \beta_{l} \wedge \alpha_{k}$.

${ }^{11}$ In order to make the equations and the statement symmetric, one might try to rescale and use both the total field strength and the twist as $\frac{1}{\sqrt{2}} G$. However, the equation of motion would then have an anomalous relative factor of $\sqrt{2}$. 
but this is zero for the same reasons that equation (3.1) holds, namely by use of the EOM and the Bianchi identity, and by the fact that the rest of the terms have high degrees. Thus,

$$
d_{G}^{2} G=0
$$

This is on-shell and is valid when the differential acts on the field strength. In the case of type II string theory, $d_{H}=d+H_{3}$ was an actual differential, i.e. $d_{H}^{2}$ was zero without necessarily acting on the RR field $F$. Does this happen in our case of M-theory?

Let us study the question one step at a time. To start, calculating $d_{G}^{2}$ gives the sum

$$
\frac{1}{2} G_{4} \wedge d+\frac{1}{2} G_{4} \wedge d
$$

i.e. $G_{4} \wedge d$. Obviously this is not zero, and so we need to modify the differential in order to have any hope at nilpotency. The problem can be traced back to the fact that $G_{4}$ has an even degree and so moving the differetnial over it does not pick a minus sign that would then cancel the other factor. Explicitly, the square gives the cross terms $d\left(G_{4} \wedge\right)+G_{4} \wedge d$, which when expanded gives $d G_{4} \wedge+G_{4} \wedge d+G_{4} \wedge d$. The first term disappears because of the Bianchi identity but the second adds to the third (instead of subtracting had $G_{4}$ been of odd degree). Thus the problem does not arise for $* G_{4}$. Note that at this stage we can see that the somewhat artificial factor of half inside the differential does not seem to matter. We will see that this is indeed the case later.

In order to get the two terms above to subtract instead of add, we need some form of grading. For that purpose, let us use the duality-symmetric total field strength introduced in [17],

$$
\mathcal{G}=v G_{4}+\widetilde{v} * G_{4}
$$

and check whether this $\mathcal{G}$ can be used as a twist to form the desired differetial. As the problem above was due to the sign in the Leibnitz rule, let us consider the corresponding rule for $\mathcal{G}$. Due to the nature of $v$ and $\widetilde{v}[17$, this is

$$
d(\mathcal{G} \wedge)=d \mathcal{G} \wedge-\mathcal{G} \wedge d
$$

Then using this Leibnitz rule to expand the expression

$$
(d \pm \mathcal{G})^{2}=d^{2} \pm d(\mathcal{G} \wedge) \pm \mathcal{G} \wedge d+\mathcal{G} \wedge \mathcal{G}
$$

gives

$$
(d \pm \mathcal{G})^{2}= \pm d \mathcal{G}+\mathcal{G} \wedge \mathcal{G}
$$


Now which sign to pick is determined simply by the vanishing of the right hand side. This happens for the minus sign ${ }^{12}$ because then the right hand side would be

$$
d \mathcal{G}-\mathcal{G} \wedge \mathcal{G}
$$

which is zero as it is just the negative of the unified equation giving the EOM and the Bianchi identity derived in [17. Then, $d-\mathcal{G}$ is indeed a differential, which we will denote by $d_{\mathcal{G}}$. At this point we can try to look for slight variations of this differential.

- Scaling: From the expression

$$
(d+n \mathcal{G})^{2}=-n d \mathcal{G}+n^{2} \mathcal{G} \wedge G
$$

we see that the constant $n$ can only be equal to one in order for the unified equation of motion to be satisfied.

- Duality: We can derive the Leibnitz rule for the dual field $* \mathcal{G}$,

$$
d(* \mathcal{G} \wedge)=d(* \mathcal{G}) \wedge+* \mathcal{G} \wedge d
$$

which we use to show that

$$
(d \pm n * \mathcal{G})^{2}= \pm n d * \mathcal{G} \wedge \pm n * \mathcal{G} \wedge d \pm n * \mathcal{G} \wedge d+n^{2} * \mathcal{G} \wedge * \mathcal{G}
$$

It is obvious then that $(d \pm n * \mathcal{G})$ is not a differetial since the terms $\pm n * \mathcal{G} \wedge d$ in (3.14) add, giving a result that cannot be zero without acting in a particular way on other forms.

So does this mean we have twisted cohomology? This suggests that one gets such a structure if one uses the rank seven field $*_{11} G_{4}$ as the dual field of the M-theory rank four field $G_{4}$. At the level of differential forms, the differential $d_{G}$ is then interpreted as a map

$$
d_{G}: \Omega^{m} \oplus \Omega^{m-3} \longrightarrow \Omega^{m+1} \oplus \Omega^{m-2},
$$

our case being $m=7$ of course. Such differentials (with one twist) were encountered in [21]. One can also form a differential of uniform degree by introducing a formal parameter $t$ of degree -3 and write ${ }^{13} d_{G_{4}}=d+t G_{4}+t^{2} * G_{4}$. The interpretation of $t$ as a periodicity generator is desirable but is not very transparent again because it is of odd degree. This

\footnotetext{
${ }^{12}$ One way this minus sign can be motivated is by saying it gives the differential $d-H$ in type IIA upon reduction (at least of the $G_{4}$-part of $\mathcal{G}$.

${ }^{13}$ We are oversimplifying as we also have to include $v$ and $\widetilde{v}$. We hope to discuss this elsewhere.
} 
shift from even to odd degrees can be obtained by suspension or by looping (see below for relevance).

Furthermore, we would like to interpret the above result at the level of twisted cohomology as the target via a generalized Chern character of some twisted generalized cohomology theory $\mathcal{M}(\bullet, G)$ or ${ }^{\tau} \mathcal{M}$, where $\tau$ is $[G]$, the class of $G$, i.e.

$$
\operatorname{ch}_{G}: \mathcal{M}(\bullet, G) \longrightarrow H^{4 k}(\bullet, G) .
$$

Note that elliptic cohomology theory can be thought of, at least heuristically, as the K-theory on the loop space, i.e. the elliptic cohomology of a space $X$ is the K-theory of $L X$. The twists of K-theory are given by its automorphism. This includes $H^{3}(X ; \mathbb{Z})$. Applying this to the loop space gives the automorphism of elliptic cohomology, by which one can twist. ${ }^{14}$ By transgression, $H^{3}(L X ; \mathbb{Z})$ gives $H^{4}(X ; \mathbb{Z})$. For the $d * G_{4}$ part, we expect the arguement to be analogous. The $H^{8}$-twist in M-theory would descend to $H^{7}$-twist in string theory. ${ }^{15}$

\subsection{Including the one-loop term}

The EOM after including the one-loop term (first introduced in [22]) is modified to

$$
d * G_{4}=-\frac{1}{2} G_{4} \wedge G_{4}+I_{8}
$$

where $I_{8}=-\frac{p_{2}-\left(p_{1} / 2\right)^{2}}{48}$ is the purely gravitational term, a polynomial in the Pontrjagin classes of the tangent bundle of the eleven dimensional spacetime $Y^{11}$.

We can still group together $G_{4}$ and its dual in the presence of $I_{8}$. For the degree four/eight combination we simply add $I_{8}$ to $d * G_{4}$ and we are dealing with precisely the $\Theta$-class studied in [19] and [8]. For the case of the degree four/seven combination, we can use the fact that $I_{8}=d X_{7}$ where $X_{7}$ is the transgression polynomial for $I_{8}$ in degree seven, and write the expressions using $* G_{4}+X_{7}$. For example,

$$
\left(d+\frac{1}{2} G_{4}\right)\left[G_{4}+\left(* G_{4}+X_{7}\right)\right]
$$

with the degree five and degree eight pieces giving respectively the Bianchi identity and the EOM upon using $d X_{7}=I_{8}$. Other formulae follow as well. We can see that when we add $I_{8}$ to the picture, it serves as an obstruction to having a twisted theory. However, if we absorb it in the defintion of the dual field as above, then we would still get a twist.

\footnotetext{
${ }^{14}$ We thank Constantin Teleman for explanations concerning this point.

${ }^{15}$ We hope to discuss this in detail elsewhere.
} 


\section{Acknowledgements}

The author thanks Igor Kriz for helpful explanations on generalized cohomology theories, Edward Witten for very interesting discussions, Varghese Mathai for very useful comments, and Arthur Greenspoon for suggestions in improving the presentation. He also thanks the organizers of the Oberwolfach mini-workshop on Gerbes, Twisted K-theory and Conformal Field Theory, the Simons Workshop in Mathematics and Physics at Stony Brook, and the Theory Division at CERN for their hospitality during the final stages of this project.

\section{References}

[1] G. Moore and E. Witten, Self-duality, Ramond-Ramond fields, and K-theory, JHEP 0005 (2000) 032, arXiv:hep-th/9912279.

[2] E. Diaconescu, G. Moore and E. Witten, $E_{8}$ gauge theory, and a derivation of K-theory from M-theory, Adv. Theor. Math. Phys. 6 (2003) 1031, arXiv:hep-th/0005090.

[3] V. Mathai and H. Sati, Some relations between twisted K-theory and $E_{8}$ gauge theory, JHEP 0403 (2004) 016, arXiv:hep-th/0312033.

[4] I. Kriz and H. Sati, M Theory, type IIA superstrings, and elliptic cohomology, Adv. Theor. Math. Phys. 8 (2004) 345, arXiv:hep-th/0404013.

[5] I. Kriz and H. Sati, Type IIB string theory, S-duality and generalized cohomology, Nucl. Phys. B715 (2005) 639, arXiv:hep-th/0410293.

[6] I. Kriz and H. Sati, Type II string theory and modularity, JHEP 08 (2005) 038, arXiv:hep-th/0501060.

[7] H. Sati, M-theory and Characteristic Classes, JHEP $08 \quad$ (2005) 020 arXiv:hep-th/0501245.

[8] H. Sati, Flux quantization and the M-theoretic characters, Nucl. Phys. B727 (2005) 461, arXiv:hep-th/0507106.

[9] R. Minasian and G. Moore, K-theory and Ramond-Ramond charge, J. High Energy Phys. 11 (1997) 002, arXiv:hep-th/9710230.

[10] J. Evslin and H. Sati, SUSY vs $E_{8}$ gauge theory in 11 dimensions, JHEP 0305 (2003) 048, arXiv:hep-th/0210090. 
[11] E. Cremmer, B. Julia, and J. Scherk, Supergravity in theory in 11 dimensions, Phys. Lett. B76 (1978) 409.

[12] H. Nicolai, P. K. Townsend, and P. van Nieuwenhuizen, Comments on elevendimensional supergravity, Lett. Nuovo Cim. 30 (1981) 315.

[13] I. Bandos, N. Berkovits and D. Sorokin, Duality-symmetric eleven-dimensional supergravity and its coupling to M-branes, Nucl. Phys. B522 (1998) 214, arXiv:hep-th/9711055.

[14] A. H. Chamseddine, Interacting supergravity in ten dimensions: The role of the six-index gauge field, Phys. Rev. D24 (1981) 3065.

[15] A. H. Chamseddine, Duality transformations in the ten-dimensional action and new superstring theories, Fortsch. Phys. 44 (1996) 507, arXiv:hep-th/9606010.

[16] I. A. Bandos, A. J. Nurmagambetov, and D. P. Sorokin, Various faces of type IIA supergravity, Nucl. Phys. B676 (2004) 189, arXiv:hep-th/0307153.

[17] E. Cremmer, B. Julia, H. Lu and C. N. Pope, Dualisation of dualities, II: Twisted self-duality of doubled fields and superdualities, Nucl.Phys. B535 (1998) 242, arXiv:hep-th/9806106.

[18] E. Witten, On Flux quantization in M-theory and the effective action, J. Geom. Phys. 22 (1997) 1, arXiv:hep-th/9609122.

[19] E. Diaconescu, D. S. Freed and G. Moore, The M-theory 3-form and E 8 gauge theory, arXiv:hep-th/0312069.

[20] D. S. Freed, K-theory in quantum field theory, in Current developments in mathematics, 41, Int. Press, Somerville, MA, 2002, arXiv:math-ph/0206031.

[21] P. Bouwknegt, K. Hannabuss, and V. Mathai, T-duality for principal torus bundles and dimensionally reduced Gysin sequences, arXiv:hep-th/0412268.

[22] M. J. Duff, J. T. Liu, and R. Minasian, Eleven dimensional origin of string/string duality: a one loop test, Nucl. Phys. B452 (1995) 261, arXiv:hep-th/9506126. 